\title{
Endovascular Repair of an Aortoesophageal Fistula and Fast-growing Thoracic Aortic Pseudoaneurysm Caused by Ingestion of a Fishbone
}

\author{
Yelee Kwon ${ }^{1}$, Pil Je Kang ${ }^{1}$, and Won chul $\mathrm{Cho}^{2}$ \\ ${ }^{1}$ Asan Medical Center \\ ${ }^{2}$ Gangneung Asan Hospital
}

April 20, 2021

\begin{abstract}
Thoracic endovascular aortic repair has been preferred over surgery for aortic lesions owing to its minimal invasiveness. Here we report the successful endovascular repair of a thoracic aortic pseudoaneurysm after esophageal perforation induced by accidental ingestion of a fishbone.
\end{abstract}

\section{Introduction}

Although rare, accidental ingestion of a fishbone leading to penetration of the esophagus is potentially fatal, and can result in complications such as major vascular trauma, significant hemorrhage, mediastinitis, and aortoesophageal fistula (AEF) ${ }^{[1]}$. Treatment of foreign body-induced AEF with aortic pseudoaneurysms should include repair of the damaged vessel and intensive use of antibiotic drugs, which still lacks standardization. We report the successful endovascular repair of a thoracic aortic pseudoaneurysm after esophageal perforation caused by accidental ingestion of a fishbone.

\section{Case report}

A 74-year-old man was admitted to our hospital for worsening dysphagia, fever, and intermittent retrosternal discomfort 7 days after he had eaten a meal containing fish. On the day of the meal, the patient had presented to the emergency department of a different hospital with dysphagia and odynophagia, and believed he had swallowed a fishbone. At that time, a laryngoscopy was performed but showed no evidence of foreign material, so the patient was discharged. He had a fever of $39.0^{\circ} \mathrm{C}$, normotension of $133 / 68 \mathrm{~mm} \mathrm{Hg}$, and tachycardia of $108 \mathrm{bpm}$ on admission to our hospital. Laboratory testing documented severe leukocytosis $($ WBC $19.1 \times$ $10^{3} / \mathrm{uL}$ ) and an increased C-reactive protein concentration (16.47 mg/dL). A computed tomography (CT) scan showed a thin foreign body in the upper third of the esophagus, penetrating the wall and almost reaching the thoracic aorta (Fig. 1A), combined with mediastinitis that included free periesophageal air bubbles and fluid collection. No clinical or radiologic signs of active bleeding from the thoracic aorta were noted at that time. An emergent esophagogastroduodenoscopy (EGD) was performed, and the fishbone fragment was removed (Fig. 1B). While there was no active bleeding, deep ulceration of the esophagus was observed (Fig. 1C). The next day, follow-up enhanced chest CT revealed a tiny pseudoaneurysm of the thoracic aorta (Fig. 2A), which had increased at the subsequent CT angiogram on the third day (Fig. 2B). Immediately after the third CT scan, thoracic endovascular aortic stent-graft placement was performed to treat the pseudoaneurysm under local anesthesia through the femoral artery. The covered portion of the graft was placed just distal to the origin of the left common carotid artery. Two days after endovascular repair, the patient had approximately a half cup-amount of hematemesis. An EGD and CT angiogram were 
immediately performed. The CT scan showed a much larger saccular outpouching lesion of the thoracic aorta, indicating that the pseudoaneurysm was growing (Fig 2C). However, the patient's vital signs were stable, and the pseudoaneurysm on the CT angiogram was lower contrast-enhanced than the aorta proper, so we decided to proceed with conservative treatment using the Sengstaken-Blakemore tube (SB tube). Four days after the procedure, we removed the SB tube and hematemesis did not reoccur. Upon admission, antibiotic treatment was administered according to the following regimen: IV cefepime $[2.0 \mathrm{~g}]$ and metronidazole $[0.5$ g] thrice daily. Six days after the intervention, the patient's temperature decreased to below $38^{\circ} \mathrm{C}$. C-reactive protein concentration started to decrease 3 days after the intervention and normalized on the 12th day postprocedure. Follow-up CT angiography showed that the pseudoaneurysm had completely disappeared 14 days after the placement of the stent (Fig. 2D). Although the patient had undergone the McKeown procedure due to consistent esophageal perforation 2 months later, AEF and pseudoaneurysm did not reoccur.

\section{Comment}

Esophageal penetration caused by accidental foreign body ingestion is uncommon, with a reported incidence of $1-4 \%{ }^{[2]}$. Additionally, AEF is rare; Nandi et al. ${ }^{[3]}$ reported that only $1 \%$ of patients had esophageal perforation caused by swallowing fish or chicken bones among 2394 cases of foreign body ingestion, and $0.1 \%$ of these patients developed AEF. Given the low rate of occurrence, it is not unexpected that there are no established treatment guidelines for AEF.

According to the article by Takeno et al., which reviewed trends of AEF management, thoracic endovascular aortic repair (TEVAR) was typically preferred over surgery for aortic lesions. In contrast, esophagectomy was preferred for esophageal lesions to remove the original infectious source ${ }^{[4]}$, as was the case for our patient. This trend might have reflected the minimal invasiveness of TEVAR compared with surgical procedures for the thoracic aorta, which needed a cardiopulmonary bypass, leading to increased bleeding risk.

Aortic pseudoaneurysm could have developed secondary to the infectious mediastinitis ${ }^{[5]}$. Although there was no clear evidence of aortic lesion at the first observation in this case (Fig 1A), a small but expanding pseudoaneurysm was observed during subsequent radiologic follow-up (Fig 2A-C). The weakness of the aortic wall attributed to an adventitial tear combined with mediastinitis might have contributed to the rapidly growing pseudoaneurysm. Therefore, if esophageal perforation is diagnosed, CT angiography should be performed as soon as possible to ensure the early detection of any vascular problems, such as aortic rupture or aneurysm formation. Moreover, close patient monitoring and surveillance should be considered, even if no significant problem is detected upon initial examination.

\section{Figures}

Figure 1. Computed tomography (A) and endoscopic (B) images demonstrate that the fishbone (arrow) penetrates the esophagus and almost reaching the aorta. (C) After removal of the fishbone, deep ulceration was observed.
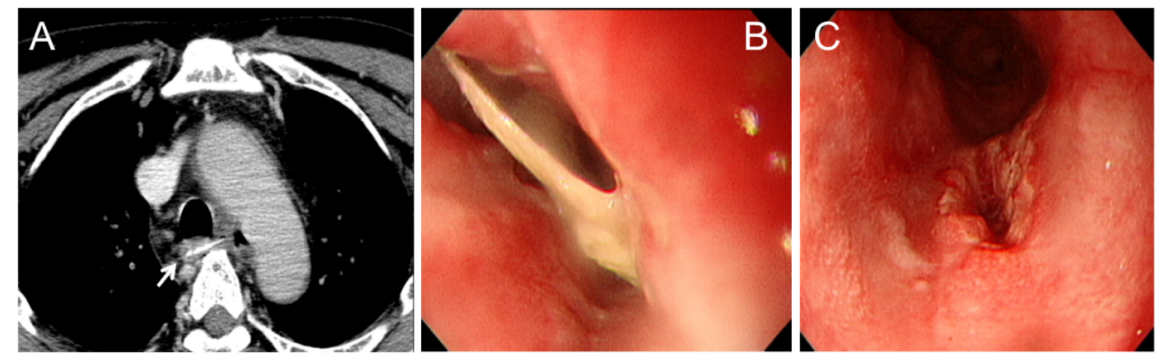

Figure 2. Follow-up computed tomography revealed tiny pseudoaneurysm the day after admission (A), growing size of the pseudoaneurysm on the third day (B), after thoracic endovascular aortic stent-graft placement showing the larger size of the pseudoaneurysm (C). The pseudoaneurysm had disappeared 14 days after the placement of the stent (D). 

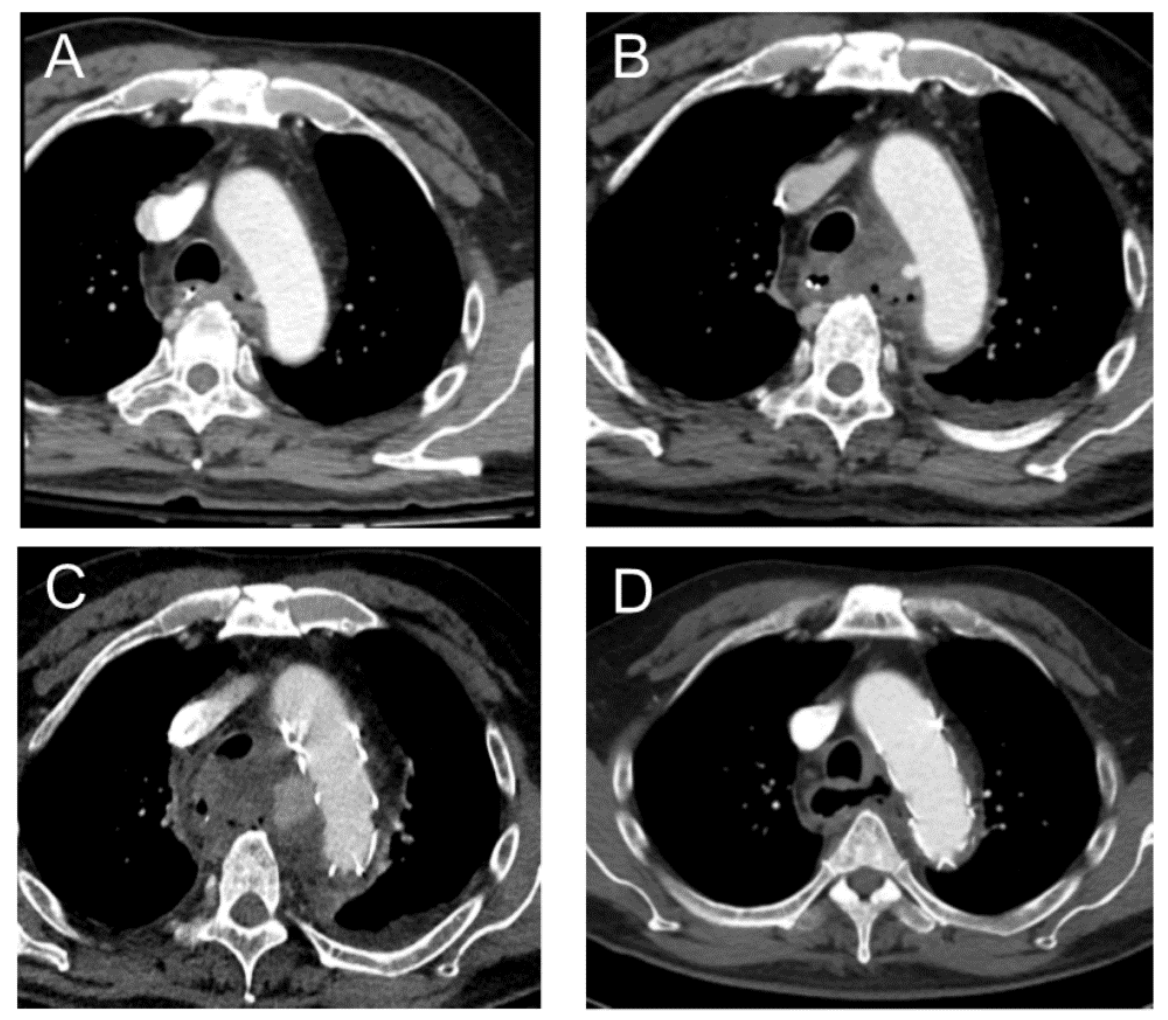

\section{References}

1. Bathla G, Teo LL, Dhanda S. Pictorial essay: complications of a swallowed fish bone. Indian J Radiol Imaging. 2011 Jan;21(1):63-68.

2. Scher RL, Tegtmeyer CJ, McLean WC. Vascular injury following foreign body perforation of the esophagus: review of the literature and report of a case. Ann Otol Rhinol Laryngol. 1990 Sep;99(9 Pt 1):698-702.

3. Nandi P, Ong GB. Foreign body in the oesophagus: review of 2394 cases. Br J Surg. 1978 Jan; 65(1):5-9 4. Takeno S, Ishii H, Nanashima A, Nakamura K. Aortoesophageal fistula: review of trends in the last decade. Surg Today. 2020 Dec;50(12):1551-1559

5. D'Costa H, Bailey F, McGavigan B, George G, Todd B (2003) Perforation of the oesophagus and aorta after eating fish: an unusual cause of chest pain. Emerg Med J. 2003 Jul;20(4):385-386 